\title{
Первопринципное исследование электронного и колебательного строений тетрагонального диарсенида кадмия
}

\author{
(С) Ю.М. Басалаев, А.В. Копытов, А.С. Поплавной, Ю.И. Полыгалов
}

Кемеровский государственный университет, 650043 Кемерово, Россия

E-mail: ymbas@mail.ru

(Получена 18 октября 2016 г. Принята к печати 15 ноября 2016 г.)

\begin{abstract}
Электронный спектр, деформационная электронная плотность и фононные частоты в центре зоны Бриллюэна тетрагонального соединения $\mathrm{CdAs}_{2}$ вычислены из первых принципов на основе метода функционала плотности. Установлено, что кристалл является непрямозонным с шириной запрещенной зоны $\sim 1$ эВ, что хорошо согласуется с известными оптическими и электрофизическими экспериментальными данными. Изучены особенности образования химической связи в кристалле, обусловленные тем, что атомы мышьяка образуют спиральные цепочки из ковалентных связей As-As, тогда как связь Cd-As является ионноковалентной. Вычисленные из первых принципов и в модели Китинга фононные частоты сопоставлены между собой и с экспериментом, выполнен анализ парциональных вкладов атомов $\mathrm{Cd}$ и $\mathrm{As}$.
\end{abstract}

DOI: $10.21883 /$ FTP.2017.06.44562.8434

\section{1. Введение}

Диарсенид кадмия $\left(\mathrm{CdAs}_{2}\right)$ синтезирован более ста лет назад $[1,2]$ и нашел широкое применение в электронике и оптоэлектронике благодаря своим структурным особенностям и уникальным свойствам [3]. В монографии [3] дан детальный обзор методам синтеза этого соединения и исследованиям его физических свойств. В первых исследованиях электрофизических свойств нелегированных кристаллов $\mathrm{CdAs}_{2}$, выращенных различными методами и всегда обладающих $n$-типом проводимости, установлены анизотропия удельного сопротивления, изотропия коэффициентов Холла, отсутствие продольного магнитосопротивления [4-7]. На этом основании сделан вывод, что поверхности постоянной энергии могут быть представлены эллипсоидами вращения, ориентированными вдоль оси 4-го порядка $c$, тогда отношение удельных сопротивлений $\rho_{a} / \rho_{c}$ равно отношению эффективных масс электронов в а и с направлениях $m_{a} / m_{c} \approx 4$. Этот результат был подтвержден исследованиями циклотронного резонанса [8], в которых для эффективных масс электронов в направлениях а и с получены значения: $m_{a}=0.58 m_{0}, m_{c}=0.15 m_{0}\left(m_{0}-\right.$ масса свободного электрона), отношение $m_{a} / m_{c}=3.87$ близко к оцененному в [5]. Значения эффективных масс дырок также оказались анизотропными и равными $m_{a}=0.346 m_{0}, m_{c}=0.094 m_{0}$. То, что эффективные массы электронов оказались больше эффективных масс дырок, не вполне обычный факт, из которого следует, что в области собственной проводимости $\mathrm{CdAs}_{2}$ будет обладать проводимостью $p$-типа. В последующих исследованиях электрофизических свойств [9-12] в широком температурном интервале $(77-620 \mathrm{~K})$, на разных образцах $n$-типа отмечается, что анизотропия удельного сопротивления незначительна, $\rho_{a} / \rho_{c} \sim 1.1-1.3$, и не изменяется в области температур 300-450 К. Различие в величинах $\rho_{a} / \rho_{c}$, полученных разными авторами, составляет от 1.1 до 4 при $300 \mathrm{~K}$, связывается в [3] как со степенью совершенства исследуемых кристаллов, так и с методиками измерений электрических и гальваномагнитных параметров. Ширина запрещенной зоны, вычисленная из температурных зависимостей $R$ и $\rho$, равна 1.14 эВ $(0 \mathrm{~K})$; из значений термоэдс вычислены без учета анизотропии эффективные массы электронов и дырок $m_{n}=0.38 m_{0}, m_{p}=0.41 m_{0}$. Эти результаты противоречат данным циклотронного резонанса [8].

Исследования энергетических параметров зонной структуры выполнялись различными оптическими методами. В работах $[5,13]$ в области длинноволнового края собственного поглощения наблюдались два поляризованных края. Один из них определяется прямыми переходами для $\mathbf{E} \| \mathbf{c}$ при энергии 1 эВ, а другой для $\mathbf{E} \| \mathbf{a}$ при 1.04 эВ, причем интенсивность поглощения при параллельной поляризации больше, чем при перпендикулярной, где $\mathbf{E}-$ вектор напряженности электрического поля волны, падающей на образец. В работе [14] край фундаментального поглощения при $300 \mathrm{~K}$ интерпретировался как непрямой с энергиями 0.995 эВ $(\mathbf{E} \| \mathbf{c})$ и 1.00 эВ $(\mathbf{E} \| \mathbf{a})$. Экспериментальному исследованию динамики кристаллической решетки посвящены работы $[15,16]$, где были получены и проанализированы оптические спектры ИК отражения и КР света на кристаллах $\mathrm{CdAs}_{2}$.

Теоретико-групповые исследования симметрии ветвей зонного спектра, а также количественные расчеты положений энергетических уровней $\mathrm{CdAs}_{2}$ по методу эмпирического псевдопотенциала впервые выполнены в [17-20], симметрия колебательных спектров изучалась в [21]. Расчет фононных спектров на основе феноменологической модели Китинга в наиболее симметричных точках и направлениях зоны Бриллюэна выполнен в [22]. Там же вычислена и плотность фононных состояний, проведено исследование инфракрасной дисперсии и спектра поляритонов. Следует обратить внимание на то обстоятельство, что эмпирические форм-факторы псевдопотенциалов, которые использовались при вычисле- 
нии зонного спектра $\mathrm{CdAs}_{2}$ в [20], относительно надежно определены для кристаллов кубической системы и для тетрагонального $\mathrm{CdAs}_{2}$, необходимо интерполировать их в область коротких длин обратной решетки, где они не малы. Кроме того, в работах [19-21] не учитывались $d$-состояния кадмия, гибридизационные эффекты с которыми влияют на валентную часть спектра.

Как видно из приведенного обзора, имеется значительная информация о параметрах зонного и колебательного спектров $\mathrm{CdAs}_{2}$. Вместе с тем в ряде работ содержатся противоречивые сведения о структуре краев энергетических зон, отсутствуют последовательные, выполненные с единых позиций теоретические вычисления зонного и фононного спектров. В представленной работе выполнены первопринципные исследования электронного строения, химической связи и решеточной динамики тетрагональной фазы кристаллов $\mathrm{CdAs}_{2}$.

\section{2. Метод расчета и оптимизация кристаллической структуры}

Расчеты электронной структуры и колебательных мод выполнялись в программном коде CRYSTAL [23] с использованием разных базисов и в различных приближениях (LDA, GGA) с гибридными функционалами (PBE, B3LYP). В результате для атомов обоих сортов был выбран полноэлектронный базис TZVP, а в качестве наиболее сопоставимого с экспериментом функционал PВЕ. Уровень сходимости фононных частот поддерживался в пределах нескольких десятых долей $\mathrm{cm}^{-1}$. При вычислении продольных $\left(v_{\mathrm{LO}}\right)$ и поперечных $\left(v_{\mathrm{TO}}\right)$ частот использовались экспериментальные значения диэлектрических констант (11.5 и 13.8) из работы [15]. Интегрирование по зоне Бриллюэна проводилось на сетке $4 \times 4 \times 4$ для расчета энергетической зонной структуры и $8 \times 8 \times 8$ для остальных вычислений.

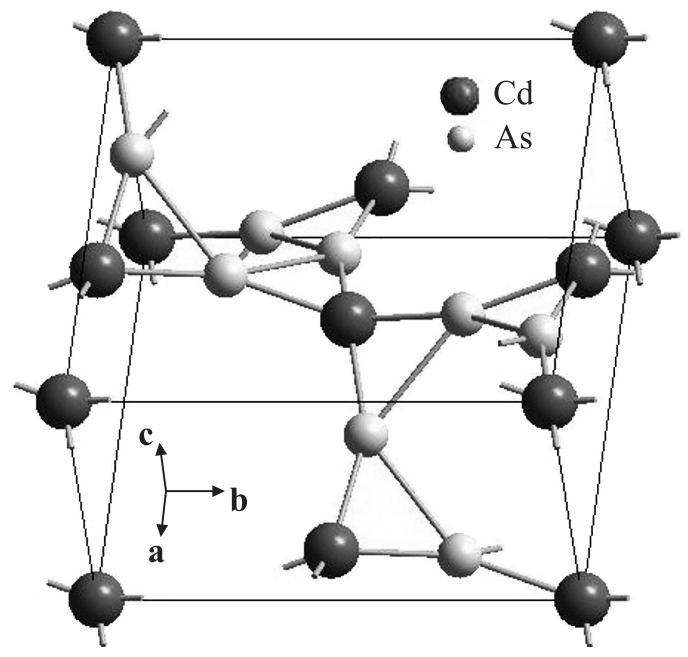

Рис. 1. Примитивная ячейка тетрагонального кристалла $\mathrm{CdAs}_{2}$.
Диарсенид кадмия кристаллизуется в тетрагональной сингонии (рис. 1) с пространственной группой $D_{4}^{10}$ (№ 98, I4 122 ). Значения структурных параметров $(a=7.954 \AA, c=4.678 \AA)$ брались из работы [24] и подвергались стандартной для кода CRYSTAL процедуре оптимизации. Оптимизированные постоянные решетки, соответствующие равновесному состоянию кристалла, имеют следующие значения: $a=7.8262 \AA$, $c=4.6624 \AA$. Координаты неэквивалентных атомов в результате оптимизации изменились несущественно по сравнению с экспериментальными данными: $\mathrm{Cd}(0,0,0)$ и As $(0.0538,0.250,0.125)$, длины связей $\mathrm{Cd}-\mathrm{As}$ и As-As оказались соответственно равными 2.6565 и $2.4646 \AA$, что согласуется с длинами связи, установленными в [24].

\section{3. Электронное строение и химическая связь}

Зона Бриллюэна тетрагонального $\mathrm{CdAs}_{2}$, отвечающая соотношению $a>c$, приведена на рис. 2. На рис. 3 представлены результаты вычислений энергетической зонной структуры $\mathrm{CdAs}_{2}$ в симметричных точках и направлениях зоны Бриллюэна, полученные с использованием программного кода CRYSTAL. Начало шкалы отсчета на этом рисунке совмещено с положением последнего заполненного уровня.

Полная ширина валентной зоны составляет величину около 12 эВ, что характерно для большинства тетрагональных диарсенидов [14], в которых валентная зона формируется преимущественно из $4 s$ и $4 p$ состояний атомов As. B $\mathrm{CdAs}_{2}$ нижняя связка валентных зон, расположенная в области от -8.5 до -12 эВ, состоит в основном из $4 s$ состояний As, а верхняя, в области от 0 до -5 эВ, - из $4 p$ состояний As. Вклады атомов $\mathrm{Cd}$ в основном локализованы вблизи -8 эВ, где на рис. 3 хорошо видна слабо дисперсная $3 d$-полоса, а также в интервале от -6 до -8 эВ, куда вносят вклад электроны $5 s$-типа.

В расчетах зонной структуры $\mathrm{CdAs}_{2}$ по методу эмпирического псевдопотенциала [19], в которых не учитывались $d$-состояния $\mathrm{Cd}$, строение валентной зоны качественно подобно представленному на рис. 3, за исключением области от -6 до -8 эВ, в которой расположена $3 d$-полоса $\mathrm{Cd}$ и имеется гибридизационный вклад $5 s$-состояний этого элемента. Ширина верхней валентной полосы, вычисленная в [19], равна 4.8 эВ, что близко к значению, полученному в этой работе, также близки по структуре и квантовому составу самые нижние валентные зоны, расположенные в области ниже -8.5 эВ. Вычисленная по методу эмпирического псевдопотенциала полная ширина валентной зоны 13.5 эВ оказалась больше значения 12 эВ, полученного в этой работе за счет использования в [19] плосковолнового базиса, который приводит к медленной сходимости энергетических уровней для низкосимметричных кристаллов со сложной элементарной ячейкой. 


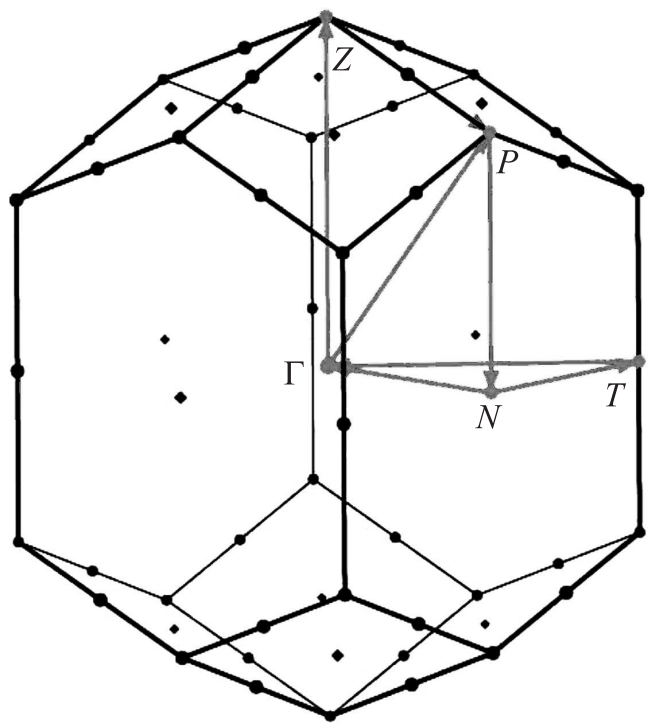

Рис. 2. Зона Бриллюэна тетрагонального кристалла $\mathrm{CdAs}_{2}$.

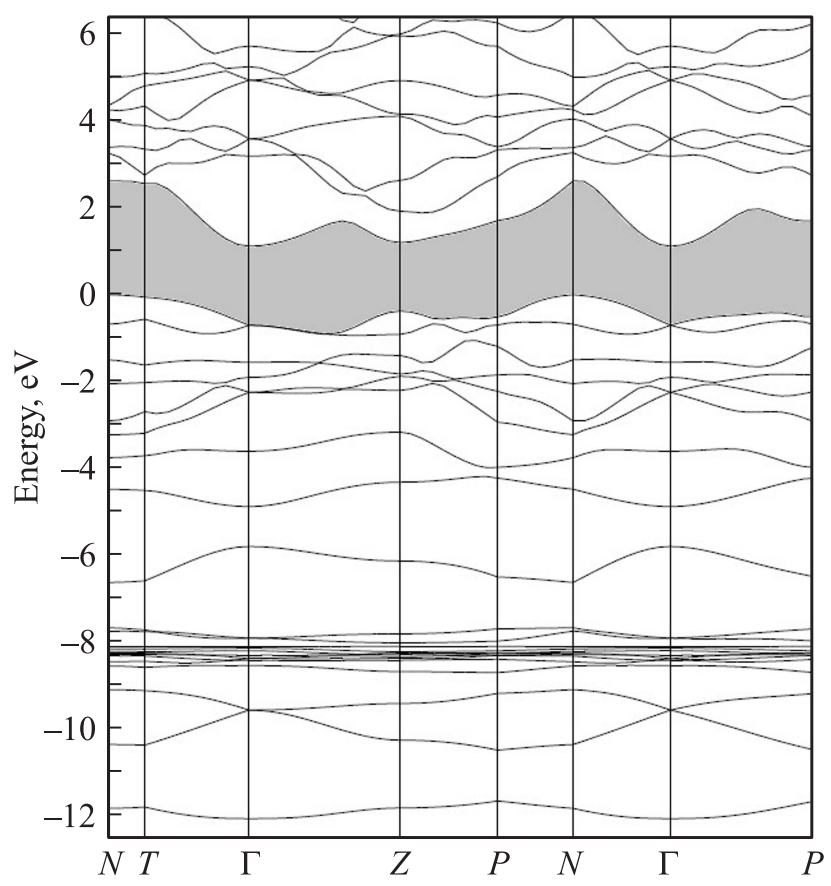

Рис. 3. Зонная структура кристалла $\mathrm{CdAs}_{2}$.

Строение краев энергетических зон - максимумов валентной зоны и минимумов зоны проводимости определяет фундаментальные физические свойства полупроводников и является достаточно чувствительным к приближениям, в которых проводятся вычисления, особенно при наличии конкурирующих экстремумов. Как видно из рис. 3, края энергетических зон $\mathrm{CdAs}_{2}$ в окрестности запрещенной зоны характеризуются наличием близких максимумов и минимумов. Относительное расположение этих экстремумов в настоящей работе и в работах [18-20] оказалось различным. Так, в настоящей работе вершина валентной зоны $\mathrm{CdAs}_{2}$ расположена в точке $N$, ближайший локальный максимум - в точке $T$, на расстоянии менее 0.1 эВ. В расчетах [19] получена обратная картина - вершина расположена в точке $T$ и на расстоянии $\sim 0.1$ эВ в точке $N$ ближайший локальный максимум. Более заметные расхождения имеют место в строении зоны проводимости. Согласно $[18,19]$, зона проводимости оказалась простой, с дном зоны в точке $T$ и ближайшими минимумами, отстоящими на расстояниях больше 0.5 эВ. Настоящий расчет дает расположение дна зоны проводимости в точке Г, имеются близкие конкурирующие минимумы (рис. 3), ближайший из которых находится в точке $Z$ на расстоянии $\sim 0.14$ эВ от дна зоны. В итоге, согласно расчетам $[18,19]$, на краю поглощения реализуется прямой разрешенный переход, согласно настоящим расчетам минимальные вычисленные непрямые переходы имеют энергии 1.00 эВ $(N \rightarrow \Gamma)$ и 1.16 эВ $(N \rightarrow Z)$. Экспериментальные исследования оптического поглощения в $\mathrm{CdAs}_{2}$ [14] показали, что края фундаментального поглощения для продольной и поперечной поляризаций определяются непрямыми переходами со значениями ширин запрещенных зон $E_{g}(\mathbf{E} \| \mathbf{c})=0.995$ эВ $(300 \mathrm{~K})$ и $E_{g}(\mathbf{E} \| \mathbf{a})=1.00$ эВ $(300 \mathrm{~K})$, достаточно близкими к вычисленным нами. В нашем расчете вычисленные минимальные непрямые переходы $N \rightarrow \Gamma(1.00$ эВ) и $N \rightarrow Z$ (1.16 эВ) согласуются как с данными [14], так и с данными электрофизических измерений [9-12]. Интенсивные прямые переходы, согласно нашему расчету, возможны в точках $Z(1.64$ эВ) и Г (1.86 эВ) и при высших энергиях в точках $P(2.58$ эВ), $N(3.01$ эВ) и $T(3.08$ эВ).

Особенности образования химической связи в кристалле $\mathrm{CdAs}_{2}$ устанавливаются по картам распределения заряда валентных электронов $\rho(\mathbf{r})$ и деформационной плотности $\Delta \rho(\mathbf{r})$. Сложность изучения электронной плотности распределения заряда обусловлена тем, что спиральные цепочки, состоящие из связанных между собой атомов (As-As), в пространстве кристаллической ячейки (рис. 1) расположены в разных плоскостях. Для визуализации распределения $\Delta \rho(\mathbf{r})$ был выполнен расчет в плоскости, содержащей атомы разного сорта, результаты представлены на рис. 4. Из рисунка видно, что связь между анионами (As-As) носит ярко выраженный ковалентный характер, о чем свидетельствует максимум $\Delta \rho(\mathbf{r})$ на середине связи As-As. Фактически заряд валентных электронов сосредоточен преимущественно именно на связях As-As, которые в пространстве кристалла образуют спиральную цепочку с чисто ковалентной связью, направленную параллельно оси с. На связи $\mathrm{Cd}-\mathrm{As}$ также имеется максимум, характеризующий ее ковалентную составляющую, смещенный от центра связи в сторону аниона примерно на четверть ее длины. При этом заряды на связях между атомами второй и последующей координационными сферами отсутствуют. В работе [19] выполнены вычисления $\rho(\mathbf{r})$ для $\mathrm{CdAs}_{2}$ по методу эмпирического псевдопотенциала, полученные результаты находятся в качественном соответствии с результатами данной работы. 
Таблица 1. Колебания кристаллической решетки $\mathrm{CdAs}_{2}$ в центре зоны Бриллюэна

\begin{tabular}{c|c|c|c|c|c|c|c}
\hline \multirow{2}{*}{ Симметрия } & \multicolumn{7}{|c}{ Частоты фононов $\nu_{\mathrm{To}} / \nu_{\mathrm{LO}}, \mathrm{cm}^{-1}$} \\
\cline { 2 - 8 } & Эксперимент $[5]$ & Эксперимент $[6]$ & Теория $[8]$ & LDA & PWGGA & PBE & B3LYP \\
\hline$\Gamma_{1}$ & & 223 & 217 & 226 & 223 & 225 & 226 \\
\hline$\Gamma_{2}$ & $83 / 88$ & & $75 / 81$ & $79 / 86$ & $79 / 85$ & $91 / 95$ & $94 / 86$ \\
& $202 / 213$ & & $213 / 217$ & $231 / 248$ & $239 / 247$ & $245 / 253$ & $245 / 248$ \\
\hline$\Gamma_{3}$ & & & 74 & 116 & 118 & 125 & 127 \\
& & & 142 & 151 & 156 & 160 & 161 \\
\hline$\Gamma_{4}$ & & 194 & 185 & 212 & 216 & 216 & 226 \\
& & 246 & 256 & 277 & 273 & 276 & 276 \\
\hline$\Gamma_{5}$ & $51 / 55$ & 53 & $59 / 69$ & $54 / 60$ & $51 / 55$ & $57 / 61$ & $59 / 61$ \\
& $120 / 126$ & 125 & $116 / 116$ & $130 / 137$ & $133 / 137$ & $136 / 140$ & $140 / 142$ \\
& $202 / 208$ & 210 & $198 / 201$ & $221 / 228$ & $223 / 226$ & $224 / 227$ & $228 / 229$ \\
& $245 / 247$ & 248 & $246 / 246$ & $260 / 262$ & $260 / 262$ & $263 / 266$ & $268 / 269$
\end{tabular}

\section{4. Вычисление фононных частот}

В табл. 1 представлены экспериментальные значения фононных частот $[15,16]$ в центре зоны Бриллюэна вместе с вычисленными в 4-х версиях функционала плотности и по феноменологической модели Китинга [22]. Как видно из таблицы, модель Китинга, содержащая 7 феноменологических параметров, дает достаточно близкие значения частот к экспериментальным, под которые велась подгонка феноменологических параметров. Первопринципные расчеты дают завышенные значения частот в верхней части фононного спектра. Это говорит о том, что традиционные версии метода функционала плотности, хорошо зарекомендовавшие себя в вычислении зонных спектров, лишь качественно правильно описывают динамическое поведение кристалла. Для по-

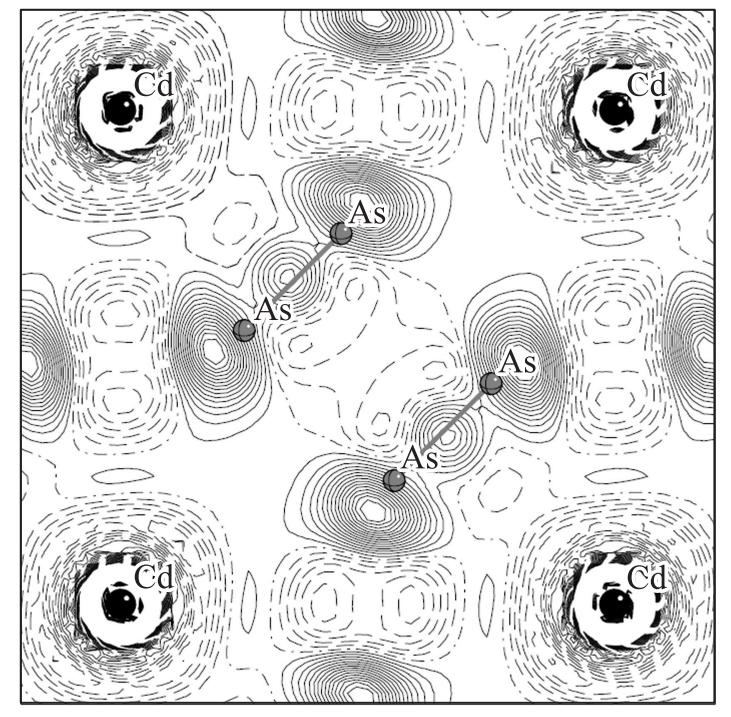

Рис. 4. Деформационная плотность распределения заряда валентных электронов кристалла $\mathrm{CdAs}_{2}$. лучения хорошего количественного согласия с экспериментом колебательных спектров необходимо специально подбирать формы обменно-корреляционного потенциала, вводить какие-либо модели дисперсионных сил типа Ван-дер-Ваальса [25]. Это несколько снижает ценность первопринципного подхода, фактически приравнивая его к феноменологическим моделям. Вместе с тем феноменологические модели имеют некоторые преимущества перед первопринципными. Так, характер химической связи, о которой в первопринципных вычислениях судят по картам электронной плотности, типа приведенной на рис. 4, устанавливается лишь качественно. Количественные характеристики - заряды ионов, заселенности связей и т. П. существенно зависят от принятой модели и не вполне однозначны. В феноменологических моделях динамики решетки параметры имеют достаточно прозрачный физико-химический смысл. Например, параметры модели Китинга, определенные для конкретных связей в [22] в единицах $10^{3}$ дин/см, имеют следующие значения: $\alpha(\mathrm{Cd}-\mathrm{As})=35.1, \alpha(\mathrm{As}-\mathrm{As})=79.3, \beta_{\mathrm{As}}^{\mathrm{CdCd}}=6.0$, $\beta_{\mathrm{Cd}}^{\mathrm{AsAs}}=5.4, \beta_{\mathrm{As}}^{\mathrm{CdAs}}=\beta_{\mathrm{As}}^{\mathrm{AsCd}}=3.0, \beta_{\mathrm{As}}^{\mathrm{AsAs}}=12.0$. Видно, что количественные характеристики связи As-As cyщественно больше, чем связи $\mathrm{Cd}-\mathrm{Cd}$. Эти численные значения могут использоваться для оценок различных физико-химических величин.

Характер колебаний устанавливается путем анализа векторов поляризации. В табл. 2 приведены в процентах вклады атомов в колебания с соответствующими частотами, вычисленными по модели Китинга [22] и CRYSTAL (PBE).

Согласно версии CRYSTAL (PBE) самые верхние оптические частоты в $\mathrm{CdAs}_{2}$ обусловлены в основном колебаниями атомов Аs. Частота $276 \mathrm{~cm}^{-1}$ с симметрией $\Gamma_{4}$ относится только к колебаниям ионов, что следует из теоретико-группового анализа. Для частоты $263 \mathrm{~cm}^{-1}$ с симметрией $\Gamma_{5}$ доля колебаний атомов As coставляет $91 \%$, а для частоты $245 \mathrm{~cm}^{-1}$ с симметрией $\Gamma_{2}$ 
Таблица 2. Вклады атомов в колебания кристаллической решетки $\mathrm{CdAs}_{2}$

\begin{tabular}{|c|c|c|c|c|c|c|}
\hline \multirow{3}{*}{ Симметрия } & \multirow{2}{*}{\multicolumn{2}{|c|}{$\begin{array}{c}\text { Частоты фононов, } \mathrm{cm}^{-1} \\
\text { Теория }\end{array}$}} & \multicolumn{4}{|c|}{ Вклады атомов, \% } \\
\hline & & & \multicolumn{2}{|c|}{ Китинг [22] } & \multicolumn{2}{|c|}{ CRYSTAL } \\
\hline & Китинг [22] & CRYSTAL & $\mathrm{Cd}$ & As & $\mathrm{Cd}$ & As \\
\hline$\Gamma_{1}$ & 217 & 225 & 0 & 100 & 0 & 100 \\
\hline$\Gamma_{2}$ & $\begin{array}{c}75 / 81 \\
213 / 217\end{array}$ & $\begin{array}{c}91 / 95 \\
245 / 253\end{array}$ & $\begin{array}{l}20 \\
38\end{array}$ & $\begin{array}{l}80 \\
62\end{array}$ & $\begin{array}{l}23 \\
34\end{array}$ & $\begin{array}{l}77 \\
66\end{array}$ \\
\hline$\Gamma_{3}$ & $\begin{array}{r}74 \\
142\end{array}$ & $\begin{array}{l}125 \\
160\end{array}$ & $\begin{array}{r}7 \\
93\end{array}$ & $\begin{array}{r}93 \\
7\end{array}$ & $\begin{array}{l}31 \\
69\end{array}$ & $\begin{array}{l}69 \\
31\end{array}$ \\
\hline$\Gamma_{4}$ & $\begin{array}{l}185 \\
256\end{array}$ & $\begin{array}{l}216 \\
276\end{array}$ & $\begin{array}{l}0 \\
0\end{array}$ & $\begin{array}{l}100 \\
100\end{array}$ & $\begin{array}{l}0 \\
0\end{array}$ & $\begin{array}{l}100 \\
100\end{array}$ \\
\hline$\Gamma_{5}$ & $\begin{array}{c}59 / 69 \\
116 / 116 \\
198 / 201 \\
246 / 246\end{array}$ & $\begin{array}{c}57 / 61 \\
136 / 140 \\
224 / 227 \\
263 / 266\end{array}$ & $\begin{array}{c}55 \\
78 \\
32 \\
2\end{array}$ & $\begin{array}{l}45 \\
22 \\
68 \\
98\end{array}$ & $\begin{array}{c}61 \\
72 \\
14 \\
9\end{array}$ & $\begin{array}{l}39 \\
28 \\
86 \\
91\end{array}$ \\
\hline
\end{tabular}

доля вклада атомов Аs уменьшается до 69\%. Следующая группа оптических частот $225 \mathrm{~cm}^{-1}$ с симметрией $\Gamma_{1}$ и $216 \mathrm{~cm}^{-1}$ с симметрией $\Gamma_{4}$ является чисто анионной по симметрии. Для оптических колебаний с частотой $224 \mathrm{~cm}^{-1}\left(\Gamma_{5}\right)$ вклад атомов As составляет 86\%. Следует отметить наличие запрещенной полосы частот между 216 и $160 \mathrm{~cm}^{-1}$. Характер формирования более низких оптических ветвей невозможно отнести к чисто массовым эффектам. Так, например, частота $160 \mathrm{~cm}^{-1}\left(\Gamma_{3}\right)$ обусловлена в основном (69\%) смещениями двух атомов $\mathrm{Cd}$ в противофазе. Для частоты $125 \mathrm{~cm}^{-1}\left(\Gamma_{3}\right)$ доли вкладов от атомов $\mathrm{Cd}$ и As взаимно поменялись. При этом интересным является симметрийное формирование частоты $125 \mathrm{~cm}^{-1}\left(\Gamma_{3}\right)$, для которой атомы каждого сорта попарно движутся в противофазе друг к другу по трем взаимно перпендикулярным направлениям. Для самых низких оптических колебаний $57 \mathrm{~cm}^{-1}\left(\Gamma_{5}\right), 91 \mathrm{~cm}^{-1}\left(\Gamma_{2}\right)$ возрастает доля вклада атомов As от 39 до $77 \%$ соответственно.

Парциальный состав колебаний, установленный в модели Китинга, качественно подобен обсужденному выше для модели CRYSTAL (PBE). Численные отличия больше для частот, значения которых различаются в этих моделях.

\section{5. Заключение}

На основе первопринципных вычислений электронного и колебательного строений тетрагонального $\mathrm{CdAs}_{2}$ установлено, что этот кристалл является непрямозонным полупроводником. Вычисленная ширина непрямой запрещенной зоны $\sim 1$ эВ находится в хорошем согласии с электрофизическими и оптическими экспериментами. Минимальные прямые переходы из валентной зоны в зону проводимости реализуются при энергиях 1.64 и
1.86 эВ. Химическая связь исследовалась путем вычисления карт деформационной электронной плотности. Заряд валентных электронов сосредоточен преимущественно на связи As-As, атомы мышьяка в пространстве кристалла образуют спиральную цепочку с ковалентной связью, направленную параллельно тетрагональной кристаллографической оси. Связь $\mathrm{Cd}-\mathrm{As}$ имеет смешанный ионно-ковалентный характер. Вычисления колебательных частот в нескольких версиях функционала плотности показали, что теоретические значения в верхней части спектра завышены по сравнению с экспериментом. Это говорит о том, что традиционные версии метода функционала плотности, хорошо зарекомендовавшие себя в вычислении электронных свойств, лишь качественно правильно описывают динамическое поведение кристалла и требуют дальнейшего совершенствования для описания межатомных сил. В этих условиях, при наличии надежных экспериментальных данных для привязки теории к эксперименту остаются полезными феноменологические модели динамики решетки. Эти модели полезны еще и тем, что силовые параметры имеют прозрачный физический смысл и могут использоваться, например, при моделировании свойств твердых тел методами молекулярной динамики или для оценки различных физических величин.

\section{Список литературы}

[1] С.Ф. Жемчужный. ЖРФХО, 34, 1281 (1905).

[2] С.Ф. Жемчужный. ЖРФХО, 45 (6), 1137 (1913).

[3] С.Ф. Маренкин, В.М. Трухан. Фосфиды, арсениды цинка и кадмия (Минск, Вараскин, 2010).

[4] Я.А. Угай, Т.А. Зюбина. Неорг. матер., 1 (6), 860 (1965).

[5] W.J. Turner, A.S. Fischler, W.E. Reese. Phys. Rev., 121 (3), 759 (1961).

[6] A.S. Fichler. Phys. Rev., 122 (2), 425 (1961). 
[7] G.A. Silvey, V.J. Lyons, V.J. Silvestry. J. Electrochem. Soc., 108 (7), 653 (1961).

[8] M.J. Stevenson Proc. Intern. Conf. Semicond. Phys., 1083 (1960).

[9] С.Ф. Маренкин, А.М. Раухман, Д.И. Пищиков, В.Б. Лазарев. Неорг. матер., 28 (9), 1813 (1992).

[10] E. Zdanowicz, W. Wojciechowski, G. Biscupski. Acta Phys. Polon. A, 77 (2-3), 383 (1990).

[11] Е. Жданович, К.Г. Лисунов. Письма ЖЭТФ, 51 (4), 207 (1990).

[12] А.М. Раухман, Д.И. Пищиков, С.Ф. Маренкин. Неорг. матер., 27 (1), 8 (1991)

[13] W.J. Turner, A.S. Fischler, W.E. Reese. Phys. Rev., 121 (3), 759 (1961)

[14] С.Ф. Маренкин, В.А. Морозова. Неорг. матер., 35 (10), 1190 (1999).

[15] I. Gregorova, J. Petzelt. Phys. Status Solidi B, 49, 271 (1972).

[16] J. Weszka, M. Balkanski, A.M. Raukhman, S.F. Marenkin. Phys. Status Solidi B, 194, 509 (1996).

[17] Ю.И. Полыгалов, А.С. Поплавной, Н.В. Кудрявцева. В кн.: Методы расчета энергетической структуры и физические свойства кристаллов (Киев, Наук. думка, 1977) c. 127.

[18] Ю.И. Полыгалов, А.С. Поплавной, В.Е. Тупицын. В кн.: Материаловедение полупроводниковых соединений группы $\mathrm{A}^{2} \mathrm{~B}^{5}$ (Воронеж, ВГУ, 1987) с. 37.

[19] Ю.И. Полыгалов, А.С. Поплавной. Изв. вузов. Физика, 32 (7), 123 (1989).

[20] В.В. Соболев, А.И. Козлов, Ю.И. Полыгалов, А.С. Поплавной, В.Е. Тупицын. Изв. АН Респ. Молдова. Физика и техника, 3 (6), 83 (1991).

[21] А.С. Поплавной, Ю.И. Полыгалов, С.И. Скачков. Изв. вузов. Физика, 18 (2), 18 (1975).

[22] А.В. Копытов, В.Н. Ткаченко. Изв. вузов. Физика, 49 (10), 327 (2006).

[23] F. Pascale, C.M. Zicovich-Wilson, F. Lopez Gejo, B. Civalleri, R. Orlando, R. Dovesi. J. Comput. Chem., 25 (6), 888 (2004).

[24] L. Gervinka, A. Hurby. Acta Crystallogr., 26 (4), 457 (1970).

[25] M. Dion, H. Rydberg, E. Schroder, D.C. Langreth, B.I. Lundqvist. Phys. Rev. Lett., 92 (24), 246401 (2004).

Редактор Г.А. Оганесян

\section{Ab-initio study of the electronic and vibrational structure of tetragonal diarsenide cadmium}

\author{
Yu.M. Basalaev, A.V. Kopytov, A.S. Poplavnoi, \\ Yu.l. Polygalov
}

\section{Kemerovo State University, 650043 Kemerovo, Russia}

Abstract Electronic spectrum, deformation electron density and the phonon frequencies in the center of the Brillouin zone tetragonal $\mathrm{CaAs}_{2}$ compound calculated from first prinsiples based on density functional theory. It was found that the crystal is not a direct-gap with a band gap of $\sim 1 \mathrm{eV}$, wich agress well with the known optical and electrophysical experimental data. The features of the formation of chemical bonds in the crystal due to the fact that the arsenic atoms form a spiral chain of covalent bonds As-As, while bond $\mathrm{Cd}-\mathrm{As}$ is ion-covalent. Calculated from first principles and the Keating model phonon frequencies are compared with each other and with experiment, the analysis of partial contributions $\mathrm{Cd}$ and As atoms. 